\title{
Over-the-Scope Clip in the Treatment of Gastrointestinal Leaks and Perforations
}

\author{
Mike T. Wei', Ji Yong Ahn² and Shai Friedland ${ }^{1}$ \\ ${ }^{1}$ Division of Gastroenterology and Hepatology, Stanford University School of Medicine, Stanford, CA, USA, ${ }^{2}$ University of Ulsan College \\ of Medicine, Asan Medical Center, Seoul, Korea
}

While perforations, postoperative fistulas, and leaks have traditionally led to surgical or interventional radiology consultation for management, the introduction of the over-the-scope clip has allowed increased therapeutic possibilities for endoscopists. While primarily limited to case reports and series, the over-the-scope clip successfully manages gastrointestinal bleeding, perforations, as well as postoperative leaks and fistulas. Retrospective studies have demonstrated a relatively high success rate and a low complication rate. Given the similarity to variceal banding equipment, the learning curve with the over-the-scope clip is rapid. However, given the higher risk of procedures involving the use of the over-the-scope clip, it is essential to obtain the scope in a stable position and grasp sufficient tissue with the cap using a grasping tool and/or suction. From our experience, while closure may be successful in lesions sized up to $3 \mathrm{~cm}$, successful outcomes are obtained for lesions sized $<1 \mathrm{~cm}$. Ultimately, given the limited available data, prospective randomized trials are needed to better evaluate the utility of the over-the-scope clip in various clinical scenarios, including fistula and perforation management. Clin Endosc 2021;54:798-804

Key Words: Fistula; Gastrointestinal leak; Over-the-scope clip; Ovesco; Perforation

\section{INTRODUCTION}

Fistulas, perforations, leaks, and refractory gastrointestinal bleeding have traditionally led to reflexive surgical or interventional radiology consultation due to the lack of endoscopic tools for adequate treatment. ${ }^{1}$ The introduction of novel devices such as endoscopic suturing systems and over-the-scope clips (OTSCs) (Ovesco Endoscopy GmbH, Tübingen, Germany) ${ }^{1}$ have provided endoscopic solutions for these challenging issues. In this review, we evaluate the use of and current data available for OTSCs. We will focus primarily on the use of OTSCs for managing leaks and perforations.

\footnotetext{
Received: October 5, 2021 Revised: November 7, 2021

Accepted: November 8, 2021

Correspondence: Mike T. Wei

Division of Gastroenterology and Hepatology, Stanford University School of Medicine, 300 Pasteur Drive, Palo Alto, CA 94304, USA

Tel: +1-650-714-2758, E-mail: mtwei@stanford.edu

ORCID: https://orcid.org/0000-0003-4756-9010

It is the invited review article.
}

(c) This is an Open Access article distributed under the terms of the Creative Commons Attribution Non-Commercial License (http://creativecommons.org/ licenses/by-nc/3.0) which permits unrestricted non-commercial use, distribution, and reproduction in any medium, provided the original work is properly cited.

\section{OTSC SYSTEM: AN OVERVIEW}

The OTSC system, approved in Europe in 2009 and by the United States Food and Drug Administration in 2010, consists of the OTSC clip, cap, thread, and hand wheel. ${ }^{2}$ The OTSC clip is installed on the cap and tethered to a thread that is controlled by the hand wheel (Fig. 1). ${ }^{3}$

The edges of the desired tissue (such as area around perforation, dehiscence, or bleeding vessel) are grasped with the cap by suction, an anchor, or a twin grasper, and the hand wheel is turned clockwise to release the clip over the area. The clip is made of nitinol and is shaped as a "bear claw"." The clip is magnetic resonance imaging conditional. ${ }^{4}$ To accommodate different applications, the OTSC cap comes in various sizes (mini, 11, 12, and 14) and depths ( 3 and $6 \mathrm{~mm}$ ).

Similarly, the clips come in the same sizes but have three types of teeth: type a (blunt), type $\mathrm{t}$ (small spikes), and type gc (elongated teeth with spikes) (Fig. 2). ${ }^{4}$ The advantages of the OTSC bear claw over traditional through-the-scope clip (TTSC) are its larger size allowing for greater area captured and greater compression force. ${ }^{3}$ The OTSC has been used to manage bleeding, leaks, and perforations as well as facilitate closure after complex polypectomy. 


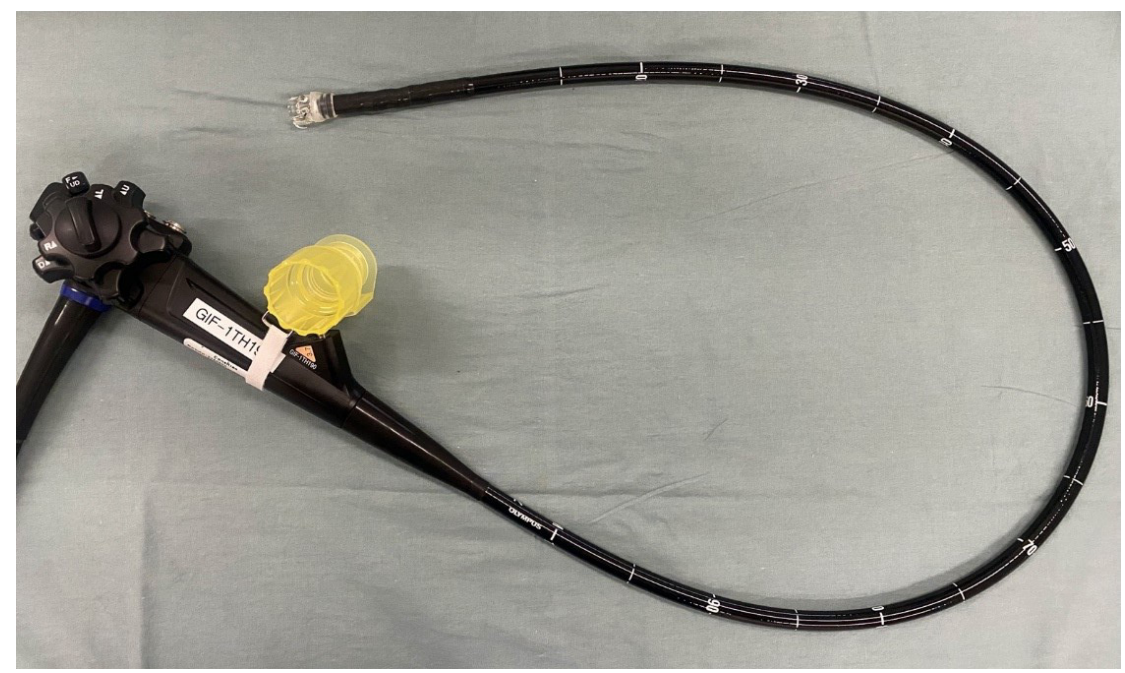

Fig. 1. Over-the-scope clip (OTSC) loaded on a therapeutic upper endoscope. The tip of the endoscope is attached to a cap loaded with the OTSC, and the biopsy channel is loaded with the hand wheel.

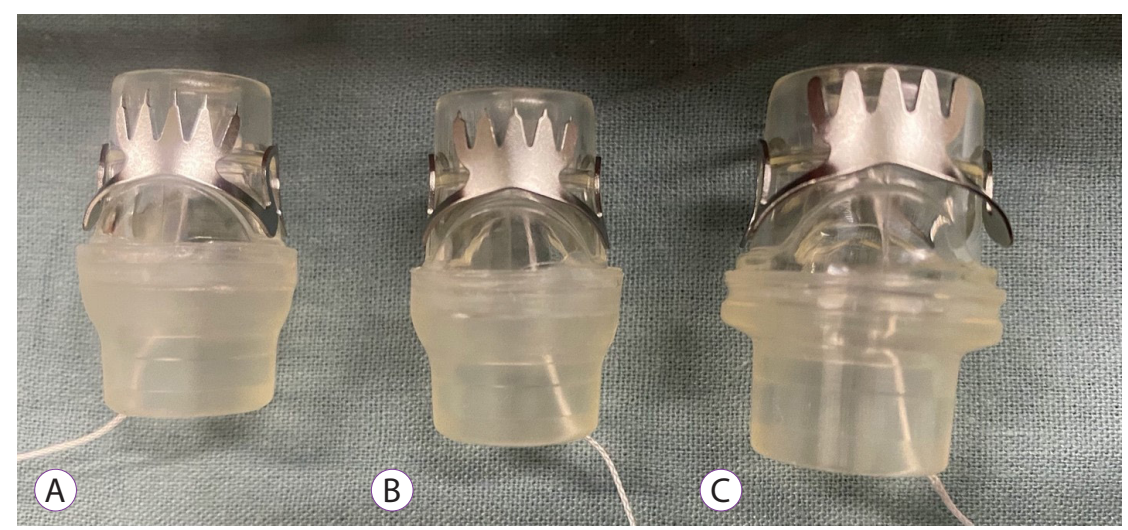

Fig. 2. Over-the-scope clip types. (A) Type gc. (B) Type t. (C) Type a.

\section{CLINICAL APPLICATIONS OF OTSC}

\section{OTSC in the management of bleeding}

In only one of two randomized trials evaluating OTSC, Schmidt et al. compared OTSC with or without epinephrine injection to standard therapy (clips or thermal therapy with epinephrine injection) for patients with recurrent peptic ulcer bleeding that occurred after initial successful hemostasis. Patients treated with OTSC experienced a lower rate of persistent bleeding than those treated with standard therapy (6.1\% vs. $42.4 \%, p=0.001)$. Of the 14 patients who received standard therapy and experienced persistent bleeding, 10 were crossed over to OTSC and achieved complete hemostasis. ${ }^{5}$ Separately, in a meta-analysis by Zhong et al. evaluating 16 studies and
769 patients with non-variceal bleeding, OTSC was successfully placed in $95.7 \%$ of lesions, and $84.2 \%$ had no re-bleeding. Twelve of the studies identified a mortality rate of $10.9 \%$ (3.9\% related to bleeding). ${ }^{6}$

\section{Evaluation of OTSC in leaks or perforations in animals}

Given the novelty of the device as well as low scenario frequency, there are currently no randomized controlled trials evaluating its use in perforations or leaks in humans. However, several trials have been conducted using animal models. Renteln et al. evaluated gastrotomy closure using TTSC versus OTSC and found 0/10 leaks for OTSC and 4/10 leaks for TTSC via a laparoscopic leak test $(p=0.09)$. On necropsy 
analysis, although TTSC was limited to the mucosa $(8 / 10)$ and submucosa $(2 / 10)$, OTSC achieved closure of the deeper layers (submucosa $7 / 10$, muscularis $2 / 10$, and serosa $1 / 10$ ). ${ }^{7}$ In addition, Renteln et al. performed a randomized controlled study in which gastrotomy performed in pigs was randomized to open surgical repair or OTSC. OTSC achieved successful closure in 18/18 cases, although 1 case required a second OTSC deployment for adequate closure. At necropsy, both surgical and OTSC closure resulted in complete closure in all cases. ${ }^{8}$ Renteln et al. also evaluated OTSC versus surgery in the closure of $10-\mathrm{mm}$ duodenal perforations and found that OTSC achieved immediate closure results comparable to those of surgical repair.

\section{Criteria for use of OTSC}

In general, several criteria are needed for successful OTSC deployment. Given the larger system diameter $(16.5 \mathrm{~mm}$ for 11; $17.5 \mathrm{~mm}$ for 11, 12; and $21 \mathrm{~mm}$ for 14), the OTSC can only be deployed if the system can successfully reach the area of interest. Strictures or significant looping can hinder OTSC use, making through-the-scope stents or TTSCs more preferable. However, other options to mitigate this may include performing balloon dilation for a stenotic area or overtube use ${ }^{10}$ to reach the area. In addition, OTSC requires that the tissue being approximated be pliable enough to be carried into the OTSC cap. Significantly fibrotic, scarred, ulcerated, or macerated tissue can preclude its successful deployment. Use of a twin grasper or anchor can be helpful. As described by Albert et al., early intervention may increase success rates compared with late intervention since this is likely related to decreased damage to the tissue and an increased ability to achieve approximation. $^{2}$

\section{Use of OTSC for managing perforations}

Case reports included a 43-year-old male with Boerhaave's alcohol ingestion who was hospitalized for 9 days prior to transfer. He had bilateral chest tubes with purulent drainage. A 12-mm OTSC was deployed over a 1.5-cm-deep ulcer in the distal esophagus. Two OTSCs were placed to ensure complete closure. An upper gastrointestinal (GI) series performed 4 days after the procedure demonstrated no leaks and the patient remained well at the 6-month follow-up. ${ }^{11}$ In another case, a 90-year-old female underwent endoscopic ultrasound and developed a $10-\mathrm{mm}$ iatrogenic perforation during the procedure. An OTSC was used, and an abdominal computed tomography with oral diatrizoate meglumine and diatrizoate sodium solution (GASTROGRAFIN ${ }^{\circledR}$ ) demonstrated no leaks. ${ }^{12}$ Finally, a 73-year-old female with a history of pelvic surgery and severe diverticulosis underwent colonoscopy and experienced a 12$\mathrm{mm}$ sigmoid perforation. This was closed with a single OTSC, and the patient was successfully discharged 48 hours later. ${ }^{13}$

\section{Use of OTSC for managing postoperative leaks and fistulas}

Surgical anastomotic dehiscence or leaks following esophagectomy and gastrectomy occurs in at least $15 \%$ of surgical cases. ${ }^{2}$ In one study of the American College of Surgeons NSQIP database at the University of Virginia between 2003 to 2006, patients treated with gastrointestinal surgeries requiring anastomosis had a leak rate of $4.8 \%$ (13.5\% for esophageal procedures). Compared with patients without leakage, patients with anastomotic leaks had a higher morbidity rate, an increased length of stay, and a higher 30-day mortality rate. ${ }^{14}$ Surgical management of anastomotic leaks can be difficult. ${ }^{15}$ There has been an increasing number of studies on endoscopic management of surgical leaks and fistulas. Therapies may include the use of TTSCs and stents. ${ }^{16}$ However, TTSCs are generally limited to smaller perforations $(<1 \mathrm{~cm}){ }^{17}$ In addition, in one retrospective review of 115 patients who received stents, anastomotic healing occurred in $70 \%$, stent dislocation occurred in $53 \%$, and in-hospital mortality in $9 \% .{ }^{18}$ As such, OTSC has been increasingly studied in terms of postoperative anastomotic leaks.

In a retrospective study by Mercky et al. who evaluated 30 patients who were treated for gastrointestinal leaks or fistulas, the majority $(60 \%)$ had gastric fistula following laparoscopic sleeve gastrectomy. Other leaks and fistulas included rectovaginal, rectovesical, urethrorectal, gastrogastric, gastrocutaneous, esophagojejunal, gastroesophageal fistula, and colorectal anastomotic leak. ${ }^{19}$ Most (30/34) clips were placed successfully, and $53 \%$ of patients were able to close the fistula or leak after the first OTSC deployment; $26.7 \%$ required additional endoscopic maneuvers (stent, clipping, and cyanoacrylate), and another $26.7 \%$ required surgery. ${ }^{19}$

Gastrogastric fistulas (GGFs) are known complications of Roux-en-Y gastric bypass and have been estimated to occur in $1.2-6 \%$ of cases, although this value may be $0-49 \%{ }^{20-22}$ While a variety of techniques have been employed to attempt endoscopic closure of GGF including TTSC and endoscopic suturing, ${ }^{23,24}$ OTSC has also been explored as a possibility. In 2016, Niland et al. presented a case series of OTSC for managing GGF. Of the 10 patients who were followed up, five (50\%) had no persistent fistula in the upper GI series. Of the nine patients who completed long-term follow-up, only three (33.3\%) had durable success. ${ }^{25}$ In comparison, in a study evaluating 95 patients undergoing endoscopic suturing or TTSC for GGF management, $0 \%$ of $>20$-mm GGFs and $32 \%$ of $<10-\mathrm{mm}$ GGFs had durable success. ${ }^{23}$ Overall, GGF is difficult to manage endoscopically, and while more exploration is needed to understand the role of OTSC, its use in larger lesions is usually 
unsuccessful.

Additional case reports regarding the use of OTSC for GI leaks include those on pancreaticogastric, ${ }^{26} \mathrm{~J} \mathrm{pouch}^{27}$ band penetration following laparoscopic gastric banding, ${ }^{28} \mathrm{~J}$ pouch-cutaneous fistula, ${ }^{27}$ esophagojejunal fistula, ${ }^{29}$ gastroesophageal fistula, ${ }^{2}$ gastrojejunal fistula, ${ }^{2}$ Crohn's and ischemic enterocutaneous fistula, ${ }^{2}$ esophagobronchial fistula, ${ }^{30}$ rectourethral and rectocutaneous fistula, ${ }^{30}$ sleeve gastrectomy leak, ${ }^{31}$ and colonic ${ }^{32}$ or gastric side closure of gastrocolocutaneous fistula related to percutaneous endoscopic gastrostomy (PEG) placement. In a retrospective analysis by Albert et al., whose cases were covered in this section, the success rate of OTSC was significantly higher when endoscopic therapy was performed within 1 week of diagnosis (100\%) versus after 4 weeks $(57 \%){ }^{2}$

\section{Closure of PEG tube site}

In a single-center case series, Heinrech et al. presented five successful closures of fistulas after PEG tube removal, of which four were related to persistent fistulas and one was due to ascites leakage. ${ }^{33}$ In another multicenter case series of 10 patients, all underwent immediate closure of the PEG fistula. One patient required a second OTSC deployment for closure of the area adjacent to the primary site. Another patient subsequently developed a leak requiring surgical closure. ${ }^{34}$ One of our patients had persistent gastric fluid drainage 3 months after PEG tube removal and was successfully managed with OTSC deployment (Fig. 3).

\section{OTSC application with endoscopic submucosal dissection}

OTSCs have also been evaluated for closure following complex polypectomy. In 2017, Tashima et al. ${ }^{35}$ evaluated OTSC deployment following endoscopic submucosal dissection (ESD) of 50 duodenal lesions ranging from 10 to $45 \mathrm{~mm}$. Using 1.4 \pm 0.5 OTSCs, the ESD site closure rate was $94.0 \%$. Surgery was required in two patients: in the first patient, closure could not be performed endoscopically and required laparoscopic suturing; in the second patient, pancreaticoduodenectomy was needed for uncontrolled bleeding. There was one case of delayed perforation related to a misplaced OTSC. This was managed using another OTSC placement. Delayed bleeding occurred in three cases but was managed using hemostatic forceps. ${ }^{35}$ Another retrospective study by Dohi et al. evaluated ESD with prophylactic closure for duodenal epithelial tumors using TTSC, OTSC, or laparoscopic closure. ${ }^{36}$ In this study, OTSC achieved the highest closure rate (98.2\%), followed by laparoscopic closure (92.3\%) and TTSC (76.9\%) $(p=0.021)$. The delayed perforation rate was the lowest for OTSC (1.8\%), although the difference was not statistically significant. In one case, delayed perforation developed in the area between the two OTSCs and required management with a polyglycolic acid (PGA) sheet. ${ }^{36}$ While the study may be hindered by selection bias, it demonstrated the ability for prophylactic closure in ESD cases.

OTSCs may also be used to manage ESD complications. In 2019, the Japan Gastrointestinal Endoscopy Society recommended TTSC as the first-line therapy for intraoperative perforation during gastric ESD. However, if unsuccessful, one can consider the use of OTSC among other methods, including endoclip-and-loop purse string closure. In delayed perforation, while surgery should be considered, endoscopic management may include OTSC or PGA sheet shielding. ${ }^{37}$ However, commentary is difficult because OTSC use in this scenario has been limited to case reports and series. ${ }^{38}$

\section{CLINICAL OUTCOMES OF OTSC}

Evaluation of the clinical outcomes of OTSC in managing leaks, fistulas, or perforations is difficult given the lack of randomized controlled trials. In an ongoing study, Dubois et al. is leading a multicenter randomized controlled trial (FISCLOSE) evaluating the OTSC Proctology clip compared with the rectal
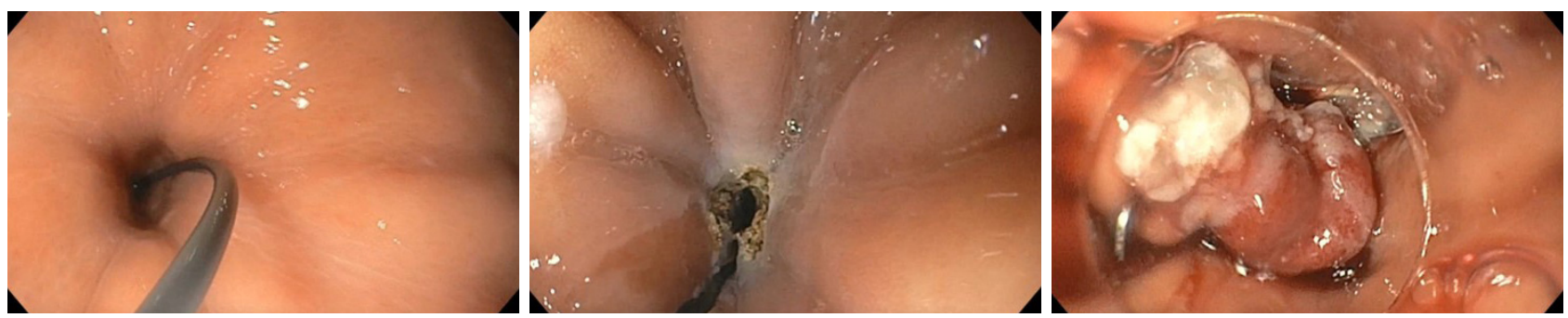

Fig. 3. Use of over-the-scope clip in the closure of percutaneous endoscopic gastrostomy tube site. 
mucosal advancement flap for complex anal fistulas. ${ }^{39}$ In one currently available randomized controlled trial, Mascagni et al. evaluated the use of the OTSC Proctology clip compared with fistulectomy and primary sphincter reconstruction for managing low trans-sphincteric anal fistulas between 2012 and 2013. In this study of 15 patients in each group, OTSC achieved a $93.3 \%$ success rate compared with a $100 \%$ success rate in the surgery group. One patient receiving OTSC required surgery, while another required OTSC removal due to post-procedure pain on day $7 .^{40}$

In addition to one randomized controlled trial, other studies are limited to case reports or small retrospective case series, ${ }^{41}$ which unfortunately have the disadvantage of selection bias, causing a possible overestimation of OTSC success rates and underestimation of complication rates. Despite limited information available in the literature, in an extensive review of 1,517 cases over 9 years (2010-2018), Kobara et al. detailed 30 studies evaluating 10 or more cases of OTSC deployment. This included OTSC used for 559 cases of hemorrhage, 388 cases of fistula, 351 cases of perforation, and 97 cases of anastomotic dehiscence. In this review, the use of OTSC achieved a 78\% success rate overall, including $85 \%$ for perforation, $85 \%$ for bleeding, $66 \%$ for anastomotic leak, and 52\% for fistula.

\section{Adverse events}

In a large review by Kobara et al., there were $26(1.7 \%)$ cases of OTSC-related complications and $9(0.6 \%)$ cases of severe complications. ${ }^{1}$ In a retrospective series by Mercky et al., 4/30 patients experienced issues with OTSC placement: clip dislodgement, fibrosis preventing clip placement, grasper anchor tearing the fistula, and gastric stenosis requiring stent placement. ${ }^{19}$ In one case of a patient who had a gastrocutaneous fistula persisting 1 year after PEG tube removal, OTSC deployment was attempted but the clip detached the following day related to significant scar tissue from months of gastric secretions. ${ }^{42}$ In another case, a 59-year-old man underwent a proximal gastrectomy for gastrointestinal stromal tumor but was found to have leakage on postoperative day 1 and underwent OTSC deployment. However, the upper GI series identified a leak on postoperative day 2 and upper endoscopy identified necrosis at the anastomosis, leading to a larger defect. The OTSC was removed, and endoscopic vacuum therapy resolved the defect. ${ }^{30}$ In a case by Mangiavillano et al., a 77-year-old female underwent endoscopic ultrasound for the evaluation of pancreatic malignancy but experienced a 15-mm full-thickness iatrogenic perforation at the posterior wall of the duodenal bulb. Upon an attempt at passing the 11-mm OTSC, the bear claw clip was accidentally deployed in the tongue and required manual removal. ${ }^{43}$

\section{What happens to the OTSC after a successful procedure?}

OTSCs may remain at the application site indefinitely or may spontaneously fall off within a few months of placement. ${ }^{44}$ Retained clips usually have no clinical consequence, although no studies have evaluated its long-term effects. However, if necessary, OTSCs can be removed after fragmentation into two pieces using either a direct-current device marketed by the manufacturer (remOVE system; Ovesco Endoscopy AG, Tübingen, Germany) or an intense application of argon plasma coagulation to the thinnest portion of the clip. In one systematic review, there were no major adverse events (perforation, bleeding requiring intervention), although removal can cause minor bleeding, superficial thermal injury, or mucosal tears. Indications for OTSC removal in the literature have included adverse events related to its placement, removal following healing, need for further intervention or biopsy, misplacement, or patient wishes. ${ }^{45}$ OTSCs that spontaneously fall off are typically excreted in the feces without difficulty, but there is one report of an OTSC becoming lodged near the anal canal and requiring removal under anesthesia. ${ }^{46}$

\section{OUR EXPERIENCE AND REFLECTIONS}

Similar to the recent American Gastroenterological Association Expert Review led by Lee et al., given the complexity of these cases, we engage the patient and family in a risk-benefit discussion prior to the procedure. ${ }^{47}$ From our experience, while closure may be successful for lesions up to $3 \mathrm{~cm}$, we found the greatest success with lesions $<1 \mathrm{~cm} .{ }^{1,36}$ For lesions $>1 \mathrm{~cm}$, we use endoscopic suturing to manage fistulas and leaks. Given the similarity to variceal banding equipment, we believe that the learning curve is rapid. However, it is important to be familiar with the equipment beforehand to reduce the risk of accidentally deploying the OTSC clip in the GI tract. ${ }^{43}$ Deep sedation or general anesthesia is often helpful, as it is important to not rush during the procedure. It is essential to obtain the scope in a stable position and bring sufficient tissue into the cap using a grasping tool and suction. Following OTSC closure of any leaks or perforations, we maintain the patient's nothing by mouth status and intravenous antibiotics until the upper GI series demonstrates a lack of contrast leak prior to advancing the diet. ${ }^{47}$

\section{CONCLUSIONS}

OTSC has been gaining traction in the management of 
bleeding, perforation, anastomotic leak, and fistula. Retrospective studies have demonstrated a relatively high success rate and low complication rate. However, prospective randomized trials are needed to better evaluate the utility of OTSC in various clinical scenarios, including fistula and perforation management. We recommend that clinicians familiarize themselves with the equipment and be deliberate during OTSC positioning and deployment to reduce the risk of unsuccessful deployment.

\title{
Conflicts of Interest
}

Mike T. Wei is consultant to Neptune Medical, Inc. and Shai Friedland is consultant to Capsovision and Intuitive Surgical.

\author{
Funding \\ None. \\ Author Contributions \\ Conceptualization: Mike T. Wei, Shai Friedland \\ Data curation: MTW, SF \\ Formal analysis: MTW, SF \\ Investigation: MTW, SF \\ Methodology: MTW, SF \\ Project administration: MTW, SF \\ Resources: MTW, SF \\ Software: MTW, SF \\ Supervision: $\mathrm{SF}$ \\ Writing-original draft: MTW, SF \\ Writing-review\&editing: MTW, Ji Yong Ahn, SF
}

\section{ORCID}

Mike T. Wei:

Ji Yong Ahn:

Shai Friedland:

\section{REFERENCES}

1. Kobara H, Mori H, Nishiyama N, et al. Over-the-scope clip system: a review of 1517 cases over 9 years. J Gastroenterol Hepatol 2019;34:22-30.

2. Albert JG, Friedrich-Rust M, Woeste G, et al. Benefit of a clipping device in use in intestinal bleeding and intestinal leakage. Gastrointest Endosc 2011;74:389-397.

3. Mennigen R, Senninger N, Laukoetter MG. Novel treatment options for perforations of the upper gastrointestinal tract: endoscopic vacuum therapy and over-the-scope clips. World J Gastroenterol 2014;20:77677776.

4. OTSC $^{\circledR}$ System Set [Internet]. Cary (NC): Ovesco Endoscopy USA, Inc:; c2021 [cited 2021 Nov 17]. Available from: https://ovesco.com/otsc-system/otsc-system-set/.

5. Schmidt A, Gölder S, Goetz M, et al. Over-the-scope clips are more effective than standard endoscopic therapy for patients with recurrent bleeding of peptic ulcers. Gastroenterology 2018;155:674-686.e6.

6. Zhong C, Tan S, Ren Y, et al. Clinical outcomes of over-the-scope-clip system for the treatment of acute upper non-variceal gastrointestinal bleeding: a systematic review and meta-analysis. BMC Gastroenterol 2019;19:225.

7. von Renteln D, Vassiliou MC, Rothstein RI. Randomized controlled trial comparing endoscopic clips and over-the-scope clips for closure of natural orifice transluminal endoscopic surgery gastrotomies. Endoscopy 2009;41:1056-1061.

8. von Renteln D, Schmidt A, Vassiliou MC, Gieselmann M, Caca K. Natural orifice transluminal endoscopic surgery gastrotomy closure with an over-the-endoscope clip: a randomized, controlled porcine study (with videos). Gastrointest Endosc 2009;70:732-739.

9. von Renteln D, Rudolph HU, Schmidt A, Vassiliou MC, Caca K. Endoscopic closure of duodenal perforations by using an over-the-scope clip: a randomized, controlled porcine study. Gastrointest Endosc 2010;71:131-138

10. Wei MT, Hwang JH, Watson RR, Park W, Friedland S. Novel rigidizing overtube for colonoscope stabilization and loop prevention (with video). Gastrointest Endosc 2021;93:740-749.

11. Al-Zahir AA, AlSaif OH, AlNaimi MM, Almomen SAM, Meshikhes A-WN. Boerhaave's syndrome: delayed management using over-thescope clip. Am J Case Rep 2019;20:816-21.

12. Grande G, Manno M, Alberghina N, et al. Quick, safe and effective repair of EUS-related duodenal perforation using over-the-scope clip system (with video). Dig Liver Dis 2016;48:1099-1100.

13. Parodi A, Repici A, Pedroni A, Blanchi S, Conio M. Endoscopic management of GI perforations with a new over-the-scope clip device (with videos). Gastrointest Endosc 2010;72:881-886.

14. Turrentine FE, Denlinger CE, Simpson VB, et al. Morbidity, mortality, cost, and survival estimates of gastrointestinal anastomotic leaks. J Am Coll Surg 2015;220:195-206.

15. Messager M, Warlaumont M, Renaud F, et al. Recent improvements in the management of esophageal anastomotic leak after surgery for cancer. Eur J Surg Oncol 2017;43:258-269.

16. Galizia G, Napolitano V, Castellano P, et al. The over-the-scope-clip (OTSC) system is effective in the treatment of chronic esophagojejunal anastomotic leakage. J Gastrointest Surg 2012;16:1585-1589.

17. Minami S, Gotoda T, Ono H, Oda I, Hamanaka H. Complete endoscopic closure of gastric perforation induced by endoscopic resection of early gastric cancer using endoclips can prevent surgery (with video). Gastrointest Endosc 2006;63:596-601.

18. Feith M, Gillen S, Schuster T, Theisen J, Friess H, Gertler R. Healing occurs in most patients that receive endoscopic stents for anastomotic leakage; dislocation remains a problem. Clin Gastroenterol Hepatol 2011;9:202-210.

19. Mercky P, Gonzalez JM, Aimore Bonin E, et al. Usefulness of overthe-scope clipping system for closing digestive fistulas. Dig Endosc 2015;27:18-24.

20. Cucchi SG, Pories WJ, MacDonald KG, Morgan EJ. Gastrogastric fistulas. A complication of divided gastric bypass surgery. Ann Surg 1995;221:387-391.

21. Carrodeguas L, Szomstein S, Soto F, et al. Management of gastrogastric fistulas after divided Roux-en-Y gastric bypass surgery for morbid obesity: analysis of 1,292 consecutive patients and review of literature. Surg Obes Relat Dis 2005;1:467-474.

22. Capella JF, Capella RF. Gastro-gastric fistulas and marginal ulcers in gastric bypass procedures for weight reduction. Obes Surg 1999;9:22-27; discussion 28.

23. Fernandez-Esparrach G, Lautz DB, Thompson CC. Endoscopic repair of gastrogastric fistula after Roux-en-Y gastric bypass: a less-invasive approach. Surg Obes Relat Dis 2010;6:282-288.

24. Merrifield BF, Lautz D, Thompson CC. Endoscopic repair of gastric leaks after Roux-en-Y gastric bypass: a less invasive approach. Gastrointest Endosc 2006;63:710-714.

25. Niland B, Brock A. Over-the-scope clip for endoscopic closure of gastrogastric fistulae. Surg Obes Relat Dis 2017;13:15-20.

26. Grande G, Caruso A, Mangiafico S, Khanna A, Conigliaro R. Overthe-scope clip in postoperative pancreatic fistula. Gastrointest Endosc 2017;85:260-261. 
27. Wei Y, Gong JF, Zhu WM. Endoscopic closure instead of surgery to close an ileal pouch fistula with the over-the-scope clip system. World J Gastrointest Endosc 2017;9:95-98.

28. Iacopini F, Di Lorenzo N, Altorio F, Schurr MO, Scozzarro A. Over-thescope clip closure of two chronic fistulas after gastric band penetration. World J Gastroenterol 2010;16:1665-1669.

29. Pohl J, Borgulya M, Lorenz D, Ell C. Endoscopic closure of postoperative esophageal leaks with a novel over-the-scope clip system. Endoscopy 2010;42:757-759.

30. Mennigen R, Colombo-Benkmann M, Senninger N, Laukoetter M. Endoscopic closure of postoperative gastrointestinal leakages and fistulas with the over-the-scope clip (OTSC). J Gastrointest Surg 2013;17:10581065.

31. Aly A, Lim HK. The use of over the scope clip (OTSC) device for sleeve gastrectomy leak. J Gastrointest Surg 2013;17:606-608.

32. Bertolini R, Meyenberger C, Sulz MC. First report of colonoscopic closure of a gastrocolocutaneous PEG migration with over-the-scope-clipsystem. World J Gastroenterol 2014;20:11439-11442.

33. Heinrich H, Gubler C, Valli PV. Over-the-scope-clip closure of long lasting gastrocutaneous fistula after percutaneous endoscopic gastrostomy tube removal in immunocompromised patients: a single center case series. World J Gastrointest Endosc 2017;9:85-90.

34. Singhal S, Changela K, Culliford A, Duddempudi S, Krishnaiah M, Anand S. Endoscopic closure of persistent gastrocutaneous fistulae, after percutaneous endoscopic gastrostomy (PEG) tube placement, using the over-the-scope-clip system. Therap Adv Gastroenterol 2015;8:182-188.

35. Tashima T, Ohata K, Sakai E, et al. Efficacy of an over-the-scope clip for preventing adverse events after duodenal endoscopic submucosal dissection: a prospective interventional study. Endoscopy 2018;50:487-496.

36. Dohi O, Yoshida N, Naito Y, et al. Efficacy and safety of endoscopic submucosal dissection using a scissors-type knife with prophylactic overthe-scope clip closure for superficial non-ampullary duodenal epithelial tumors. Dig Endosc 2020;32:904-913.

37. Yamamoto Y, Kikuchi D, Nagami Y, et al. Management of adverse events related to endoscopic resection of upper gastrointestinal neoplasms: review of the literature and recommendations from experts. Dig Endosc 2019;31(Suppl 1):4-20.

38. Nishiyama N, Mori H, Kobara H, et al. Efficacy and safety of over-thescope clip: including complications after endoscopic submucosal dissection. World J Gastroenterol 2013;19:2752-2760.

39. Dubois A, Carrier G, Pereira B, et al. Therapeutic management of complex anal fistulas by installing a nitinol closure clip: study protocol of a multicentric randomised controlled trial--FISCLOSE. BMJ Open 2015;5:e009884.

40. Mascagni D, Pironi D, Grimaldi G, et al. OTSC ${ }^{\circledR}$ Proctology vs. fistulectomy and primary sphincter reconstruction as a treatment for low trans-sphincteric anal fistula in a randomized controlled pilot trial. Minerva Chir 2019;74:1-6.

41. Mizrahi I, Eltawil R, Haim N, et al. The clinical utility of over-the-scope clip for the treatment of gastrointestinal defects. J Gastrointest Surg 2016;20:1942-1949.

42. Brent LH, Miyawaki T, Everson MP, Butler JL. Interleukin 2 production by a marmoset B cell line. Eur J Immunol 1990;20:1125-1129.

43. Mangiavillano B, Morandi E, Masci E. Accidental endoscopic piercing of the tongue with an Ovesco clip. Endoscopy 2012;44(Suppl 2 UCT$\mathrm{N})$ :E221.

44. Hu J, Yang Y, Ge N, et al. Long-term assessment of over-the-scope clip (OTSC) behavior after gastric application. Minim Invasive Ther Allied Technol 2020;29:86-89.

45. Ou YH, Kong WF, Li LF, et al. Methods for endoscopic removal of over-the-scope clip: a systematic review. Can J Gastroenterol Hepatol 2020;2020:5716981.

46. Khalid S, Satiya J, Abbass A, Parasher G, Castresana D. Migration of over-the-scope clip resulting in anal pain and obstructed defecation. Cureus 2020;12:e7572.

47. Lee JH, Kedia P, Stavropoulos SN, Carr-Locke D. AGA clinical practice update on endoscopic management of perforations in gastrointestinal tract: expert review. Clin Gastroenterol Hepatol 2021;19:2252-2261.e2. 